\title{
Desempenho e sobrevivência de alevinos de black bass (Micropterus salmoides, Lacepède 1802), submetidos ao condicionamento alimentar, utilizando diferentes patês protéicos
}

\author{
Black bass (Micropterus salmoides, Lacepède 1802) fingerlings \\ performance and survival, submitted to the alimentary conditioning, \\ using different proteics pâtés
}

\author{
Aldi Feiden ${ }^{1 *}$; Eduardo Ferrarii; Wilson Rogério Boscolo ${ }^{3}$; Marcio Freitag ${ }^{2}$; \\ Anderson Coldebella ${ }^{4}$; Luciano Hinnah ${ }^{2}$; Arcangelo Augusto Signor ${ }^{5}$
}

Resumo

\begin{abstract}
O objetivo deste trabalho foi avaliar a utilização de rações artificiais com diferentes patês protéicos incorporados na dieta de alevinos de black bass (Micropterus salmoides). Foram utilizados 320 alevinos, com peso e comprimento inicial médio de $0,57 \pm 0,1 \mathrm{~g}$ e $3,61 \pm 0,21 \mathrm{~cm}$, respectivamente. Os peixes foram distribuídos em um delineamento inteiramente casualizado em 32 caixas de polipropileno de 310 litros, com 4 tratamentos e 8 repetições. Utilizou-se uma ração referência (RR) com 38\% PB como base da dieta, sendo adicionada patês protéicos (PP) referente a cada tratamento, compostos por coração bovino (CB), sardinha eviscerada (SE), farinha de peixe chilena (FP) e filé de tilápia (FT). Durante o treinamento alimentar foi observado boa aceitação as dietas artificiais, com melhores resultados de desempenho para os tratamentos com FP e FT.
\end{abstract}

Palavras-chave: Alimentos, condicionamento alimentar, pâtés protéicos, black bass

\begin{abstract}
The aim of this study was to evaluate the use of artificial rations with different proteics pâtés incorporated in the black bass (Micropterus salmoides) fingerlings diet. 320 fingerlings, with initial average weight of $0,57 \pm 0,1 \mathrm{~g}$ and lenght of $3,61 \pm 0,21 \mathrm{~cm}$, respectively were used. The fish were distributed in an entirely casualized delineation in 32 polypropylene boxes of 310 liters, with 4 treatments and 8 repetitions. One reference ration (RR) with $38 \% \mathrm{~PB}$ as diet base was used, being added to each treatment proteics pâtés (PP), composites of bovine heart (CB), eviscerate sardine (SE), Chilean fish flour (FP) and tilapia fillet (FT). During the alimentary training good acceptance of the artificial diets was observed, with better performance results for the treatments with FP and FT.

Key words: Foods, alimentary conditioning, proteics pâtés, black bass
\end{abstract}

1 Engenheiro Agrônomo, Professor Adjunto da Universidade Estadual do Oeste do Paraná - UNIOESTE - Campus Toledo/PR, Email; aldi@unioeste.br

2 Engenheiro de Pesca, - UNIOESTE - Campus Toledo/PR.

3 Zootecnista, Professor Adjunto da Universidade Estadual do Oeste do Paraná - UNIOESTE - Campus Toledo/PR.

4 Mestre em Engenharia Agrícola pela Universidade Estadual do Oeste do Paraná - UNIOESTE - Campus Toledo/PR.

5 Engenheiro de Pesca, Mestrando em Zootecnia - UNIOESTE/Marechal Candido do Rondon. 


\section{Introdução}

O "Largemouth Bass" é um teleósteo de água doce, pertencente à ordem Perciformes, a família Centrarchidae, o gênero Micropterus, e a espécie Micropterus salmoides (Lacepède, 1802), é conhecida no Brasil como black bass.

Como a maioria dos peixes carnívoros, o black bass é um predador com hábito alimentar onívoro, alimentando-se de tudo que estiver a seu alcance. Em geral, sua dieta no ambiente natural é composta por pequenos peixes, crustáceos, insetos e zooplâncton nas primeiras fases de crescimento (alevinos e juvenis). Portanto, o principal fator que influencia sua dieta é a disponibilidade de alimentos, que varia de acordo com as diferentes épocas do ano (DAVIS; LOCK, 1997). Estudos sobre a alimentação e nutrição de espécies de peixes, subsidiam a sua criação em sistemas manejados intensivamente com o uso de rações completas, eliminando assim a necessidade da utilização de espécies forrageiras para sua alimentação, a exemplo do que ocorre na América do Norte (LEYVA, 1998; LOVSHIN; RUSHING, 1989) a partir do desenvolvimento de técnicas de treinamento alimentar (KUBITZA; LOVSHIN; LOVELL, 1997; KUBITZA; LOVSHIN 1997a; LOVSHIN; RUSHING 1989), contribuindo para otimização dos custos de produção desta espécie.

O treino alimentar de juvenis de peixes carnívoros, visando a mudança na dieta alimentar, passando de alimento natural (vivo) à alimento artificial (ração), baseia-se em remover os peixes dos ambientes naturais, privando-os totalmente da alimentação natural, estocá-los em viveiros ou tanques artificiais em alta densidade, fornecendo-lhes freqüentemente alimento preparado com alta palatabilidade em intervalos de tempo regulares. Em um ótimo treinamento alimentar, cerca de 80 a $90 \%$ dos indivíduos estocados originalmente, devem estar aptos a aceitar dietas com alimento artificial. Estudos relatam que proles oriundas de segundas ou terceiras gerações de peixes adaptados à dieta artificial, podem ser mais facilmente treinados do que aqueles peixes selvagens, o que indica uma certa domesticação. Contudo, a duração mínima do treinamento alimentar observada para o black bass é de duas semanas (TIDWELL; COYLE; WOODS, 2000). Kubitza (1995a), afirma que existem fatores de fundamental importância para o sucesso do condicionamento alimentar, como aspecto atrativo do alimento, textura adequada para a espécie, palatabilidade e estratégias de condicionamento. Segundo Kubitza e Lovshin (1997a), uma transição gradual dos ingredientes na dieta utilizada é mais eficiente do que uma transição gradual do alimento utilizado para o condicionamento de juvenis de black bass, a partir do "krill" (Euphausia sp) desidratado e congelado para ração artificial seca. Contudo, o processo de transição alimentar da ração semi-úmida para ração seca é eficaz no condicionamento alimentar do smallmouth bass (ANDERSON, 1974; LOVSHIN; RUSHING, 1989; WILLIS; FLICKINGER, 1981, 1982).

Várias pesquisas constataram que as espécies carnívoras em geral, preferem ambientes com baixa luminosidade, influenciando principalmente na taxa de mortalidade e conforto dos animais (LUZ et al., 2002). Kubitza e Lovshin (1997b), realizando trabalho de condicionamento alimentar de M. salmoides, relatam que indivíduos com 1,4g aceitaram melhor o alimento inerte do que indivíduos com peso de $0,2 \mathrm{~g}$. De maneira geral, a maioria das espécies de peixes carnívoras, podem ser condicionadas a aceitar alimento inerte (ração) como principal componente da dieta (CAVERO et al., 2003; GUERRERO ALVARADO, 2003; KUBITZA 1995a, 1995b; LUZ et al., 2002; MOURA, 1998; MOURA; KUTIZA; CYRINO, 2000).

Segundo El-Sayed (1999) a farinha de peixe é um alimento com alto valor protéico, boa fonte de ácidos graxos essenciais, energia digestível, minerais e vitaminas. Em contrapartida, Boscolo; Hayashi e Meurer (2002) destacam a existência de dificuldades em conseguir farinhas nacionais de boa qualidade para o uso na alimentação dos peixes. No entanto, a produção de farinha de peixe é aproximadamente 
$35 \%$ do pescado total capturado (TACON; DOMINI, 1999) e futuramente sua produção não será suficiente para atender a demanda mundial, visto que não só a aqüicultura, mas na criação de outros animais domésticos utiliza-se grande quantidade deste alimento em rações.

Pesquisas utilizando componentes bovinos, como parte da dieta durante o treinamento alimentar de espécies carnívoras, como coração, indicam que estes ingredientes são boas alternativas para os produtores de espécies de peixes carnívoros diminuírem os custos de produção (GUERRERO ALVARADO, 2003; LUZ et al., 2002). Contudo, um dos compostos mais eficientes que são utilizados durante o treinamento alimentar de espécies carnívoras, como alimento atrator de alta palatabilidade, é o filé de peixe ou peixes inteiros moídos (CYRINO; KUBITZA, 2003; KUBITZA, 1995b; KUBITZA; LOVSHIN 1997b; MOURA; KUTIZA; CYRINO, 2000; STOREBAKKEN, 1998). A sardinha verdadeira é uma das principais espécies capturadas pela indústria pesqueira da região sudeste e sul do país, é utilizada principalmente como alimento humano, mas também é utilizada como complemento de diversas rações de animais (SOMMER, 1998).

O presente trabalho teve por objetivo avaliar a efetividade do treinamento alimentar e o desempenho do Black bass (M. salmoides), utilizando quatro diferentes patês protéicos na dieta durante o condicionamento alimentar.

\section{Material e Métodos}

O presente trabalho foi realizado no Laboratório de Aqüicultura da Universidade Estadual do Oeste do Paraná, UNIOESTE, Campus de Toledo, por um período de 35 dias divididos em cinco semanas. Foram utilizados 320 alevinos, com peso e comprimento inicial médio de $0,57 \pm 0,1 \mathrm{~g}$ e $3,61 \pm 0,21 \mathrm{~cm}$, respectivamente, distribuídos em 32 caixas redondas de polipropileno de coloração azul, com capacidade para 310 litros, sendo considerado uma unidade experimental uma caixa contendo 10 alevinos em densidade inicial de 33 individuos $/ \mathrm{m}^{3}$. O delineamento experimental foi inteiramente casualizado, com quatro tratamentos e oito repetições. A renovação de água manteve-se constante, a uma taxa de $30 \%$ ao dia, sem aeração. Em cada tanque experimental, foram colocadas macrófitas aquáticas flutuantes, da espécie Pistia stratiotes, visando à diminuição da luminosidade, e para evitar bruscas variações de temperatura da coluna d'água, além de gerar uma maior semelhança com o ambiente natural, visto que as unidades experimentais localizavam-se dentro de uma estufa de $75 \mathrm{~m}^{2}$ (15m x 5m), a qual fornecia as condições climáticas naturais aos indivíduos (fotoperíodo, temperatura ambiente e luminosidade). Para evitar a cobertura total da lâmina d'água pelas macrófitas aquáticas, foi colocado em cada caixa, um flutuador vazado confeccionado com isopor (10 x $20 \mathrm{~cm}$ ), o qual auxiliava no manejo alimentar efetuado através deste flutuador, além de permitir a entrada de luz natural na caixa.

As variáveis físico-químicas como $\mathrm{pH}$, oxigênio dissolvido e condutividade elétrica foram mensurados semanalmente, enquanto a temperatura foi monitorada diariamente às $8,12,15$ e 19 horas.

Os alevinos de cada tratamento foram submetidos ao condicionamento alimentar, baseado na substituição parcial e progressiva dos patês protéicos contidos na dieta semi-úmida, por ração seca. As porcentagens das misturas (ração referência + patê protéico) utilizadas no condicionamento alimentar e os dias de administração das mesmas estão apresentadas na Tabela 1 e seguem metodologia semelhante à descrita por Kubitza e Lovshin (1997a). Cada tratamento utilizava um patê protéico de origem animal como base, sendo coração bovino, sardinha eviscerada, farinha de peixe chilena e filé de tilápia. 
Feiden, A. et al.

Tabela 1. Metodologia utilizada na dieta oferecida aos alevinos de M. salmoides, durante o condicionamento alimentar nos diferentes tratamentos

\begin{tabular}{lcccccccccc}
\hline \multirow{2}{*}{ Tratamentos } & \multicolumn{2}{c}{$1^{\circ}$ Semana } & \multicolumn{2}{c}{$2^{\circ}$ Semana } & \multicolumn{2}{c}{$3^{\circ}$ Semana } & \multicolumn{2}{c}{$4^{\circ}$ Semana } & \multicolumn{2}{c}{$5^{\circ}$ Semana } \\
\cline { 2 - 10 } & $\%$ PP* & $\%$ RR** & $\%$ PP & $\%$ RR & $\%$ PP & $\%$ RR & $\%$ PP & $\%$ RR & $\%$ PP & $\%$ RR \\
\hline CB & 100 & 0 & 75 & 25 & 50 & 50 & 25 & 75 & 0 & 100 \\
SE & 100 & 0 & 75 & 25 & 50 & 50 & 25 & 75 & 0 & 100 \\
FP & 100 & 0 & 75 & 25 & 50 & 50 & 25 & 75 & 0 & 100 \\
FT & 100 & 0 & 75 & 25 & 50 & 50 & 25 & 75 & 0 & 100 \\
\hline
\end{tabular}

*PP = Patê Protéico utilizado em cada tratamento; **RR = Ração Referência.

Tratamentos: $\mathrm{CB}=$ Coração Bovino; $\mathrm{SE}=$ Sardinha Eviscerada; FP = Farinha de Peixe Chilena; FT = Filé de Tilápia

O arraçoamento dos indivíduos foi efetuado quatro vezes ao dia, às 8:00, 11:10, 14:20 e 19:30 h, fornecendo alimento inerte a uma taxa de 20, 15, 10 , 8 e $8 \%$ do peso vivo ao dia, respectivamente para a primeira à quinta semanas. Esta taxa de arraçoamento foi diminuída à medida que foram observadas sobras de ração no fundo das caixas, sendo corrigidas semanalmente. A limpeza do fundo das caixas foi efetuada a cada dois dias, retirando- se sobras de ração e eventuais peixes mortos. Quando constatada a morte de peixes, estes foram retirados imediatamente, sem a reposição dos mesmos.

A ração referência inerte utilizada no treinamento alimentar dos alevinos de M. salmoides, foi peletizada (Tabela 2). O diâmetro inicial dos péletes empregado neste trabalho foi de $1,5 \mathrm{~mm}$ para a primeira e segunda semana, aumentando para $2,5 \mathrm{~mm}$ na terceira e quarta semana, e $3,5 \mathrm{~mm}$ na quinta semana.

Tabela 2. Composição percentual e química da ração referência utilizada no treinamento alimentar de black bass (M. salmoides)

\begin{tabular}{|c|c|c|c|}
\hline Ingredientes & $\%$ & Nutrientes & \\
\hline Antioxidante (BHT) & 0,02 & Energia digestível $(\mathrm{kcal} / \mathrm{kg})^{2}$ & 3600 \\
\hline Calcário calcitico & 0,61 & Proteína bruta $(\%)^{2}$ & 38,0 \\
\hline Fosfato bicálcico & 1,46 & Cálcio (\%) & 3,00 \\
\hline Farelo de soja & 27,66 & Fibra bruta $(\%)$ & 1,68 \\
\hline Farinha de peixe & 30,23 & Fósforo total (\%) & 1,56 \\
\hline Farinha de vísceras de aves & 12,50 & Extrato etéreo (\%) & 17,56 \\
\hline Milho & 17,51 & Ácido linoléico (\%) & 5,59 \\
\hline Óleo de soja & 8,52 & Lisina $(\%)$ & 2,42 \\
\hline Suplemento $(\text { Min }+ \text { Vit })^{1}$ & 1,00 & Metionina + cistina $(\%)$ & 1,55 \\
\hline Sal & 0,50 & & \\
\hline
\end{tabular}

${ }^{1}$ Níveis de garantia por quilograma do produto: Vit. A, 500.000UI; Vit. D, 200.000UI; Vit. E, 5.000mg; Vit. K3, 1.000mg; Vit. B1, 1.500mg; Vit. B2, 1.500mg; Vit. B6, 1.500mg; Vit. B12, 4.000mg; Ác. Fólico, 500mg; Pantotenato Ca, 4.000mg; Vit. C, 15.000mg; Biotina, 50mg; Inositol, 10.000; Nicotinamida, 7.000; Colina, 40.000mg; Co, 10mg; Cu, 500mg; Fe, 5.000mg; I, 50mg; Mn, 1500mg; Se, 10mg; Zn, 5.000mg.

${ }^{2}$ Valores de energia e proteína digestíveis propostos por Boscolo; Hayashi e Meurer (2002). 
A matéria-prima para produção dos patês protéicos de coração bovino, farinha de peixe chilena e sardinha eviscerada foram adquiridas in natura, trituradas e em seguida, efetuada uma pré-secagem em estufa a $55{ }^{\circ} \mathrm{C}$ com ventilação forçada por 12 horas, visando a retirada do excesso de umidade. Após este processo, foi produzida uma quantidade de patê protéico peletizado suficiente para a primeira semana de treinamento alimentar para todos os tratamentos, sendo armazenados sob refrigeração (7 ${ }^{\circ} \mathrm{C}$ ) e oferecidos aos animais com alta taxa de umidade. Na elaboração do patê protéico de farinha de peixe, foi acrescentado água aquecida a $50{ }^{\circ} \mathrm{C}$ para a peletização, até conseguir uma consistência semelhante aos demais patês (adicionando cerca de $30 \%$ de água), não havendo posterior secagem, e demais procedimentos idênticos aos citados anteriormente.

Durante cada biometria, foi realizada também a limpeza e renovação total da água das caixas, sendo que os alevinos não eram alimentados neste dia, devido ao stress causado pelo manuseio durante a biometria.

A produção do alimento inerte utilizado na dieta ração referencia mais patê protéico foi efetuada a cada semana, conforme a quantidade necessária de cada ingrediente a ser usado, adicionando-se a quantidade necessária em cada semana de ração referencia seca e triturada, patê protéico (Tabela 1), e aproximadamente $20 \%$ de água aquecida a $50{ }^{\circ} \mathrm{C}$. Após a homogeneização dos ingredientes, estes por sua vez foram peletizados, e em seguida, retirado o excesso de umidade com a utilização de uma estufa de ventilação forçada, durante aproximadamente duas horas. Após a retirada do excesso de umidade, a ração foi oferecida aos alevinos.

Ao final do período experimental os peixes foram mantidos em jejum por 12 horas para total esvaziamento do trato digestório, posteriormente os peixes foram capturados, contados e pesados, para posteriores cálculos de ganho de peso, ganho de peso diário, conversão alimentar aparente, taxa de crescimento específico e sobrevivência. Além das variáveis descritas anteriormente, ao longo do trabalho foram realizadas observações sobre o comportamento dos peixes quanto à ocorrência de agressões, territorialismo, adaptação à dieta e competição por alimento.

Os dados obtidos foram submetidos à análise de variância ao nível de 5 \% de significância, e em caso de diferenças foi aplicado o teste de Duncan, através do programa estatístico SAEG (Sistema de Análises Estatísticas e Genéticas) (UNIVERSIDADE FEDERAL DE VIÇOSA - UFV, 1997).

\section{Resultados e Discussão}

Os valores médios das variáveis físico-químicas de temperatura média observada em todos os tratamentos, nos horários pré definidos de $8,12,15 \mathrm{e}$ $19 \mathrm{~h}$, foram $22,32 \pm 1,99,22,38 \pm 1,98,22,88 \pm 1,77 \mathrm{e}$ $22,75 \pm 1,85^{\circ} \mathrm{C}$, respectivamente. Os valores médios de condutividade elétrica, oxigênio dissolvido e $\mathrm{pH}$ foram de $99,35 \pm 4,33 \mathrm{mS} / \mathrm{cm}, 4,15 \pm 0,5 \mathrm{mg} / \mathrm{L}$ e $7,05 \pm 0,32$, respectivamente. Houve um aumento gradativo da temperatura da água, do início ao fim do experimento $\left(18\right.$ à $23{ }^{\circ} \mathrm{C}$ ). Porém, as variações encontradas principalmente nos valores da temperatura da água, além de pequenas variações no $\mathrm{pH}$, oxigênio dissolvido e condutividade da água, ocorrem dentro da amplitude considerada ótima para o conforto da espécie (M. salmoides) (TIDWELL; COYLE; WOODS, 2000), e para a maioria das espécies de peixes tropicais (BOYD, 1990).

$\mathrm{Na}$ Tabela 3 estão apresentados os valores médios das variáveis ganho de peso, ganho de peso diário, taxa de crescimento especifico, conversão alimentar aparente e sobrevivência observados ao final do condicionamento alimentar dos alevinos de black bass. 
Tabela 3. Valores médios de desempenho de alevinos de black bass submetidos ao condicionamento alimentar utilizando diferentes patês protéicos

\begin{tabular}{lccccc}
\hline \multirow{2}{*}{ Variáveis } & \multicolumn{4}{c}{ Tratamentos } & \multirow{2}{*}{ CV (\%) } \\
\cline { 2 - 5 } & CB & SE & FP & FT & 14,06 \\
Ganho de peso (GP) (g) & $4,58 \mathrm{~b}$ & $4,71 \mathrm{~b}$ & $5,79 \mathrm{a}$ & $5,35 \mathrm{ab}$ & 14,06 \\
Ganho de peso diário (GD) (g/dia) & $0,15 \mathrm{~b}$ & $0,15 \mathrm{~b}$ & $0,19 \mathrm{a}$ & $0,17 \mathrm{ab}$ & 7,13 \\
Taxa de crescimento específico (TC) & $6,74 \mathrm{~b}$ & $6,44 \mathrm{~b}$ & $7,29 \mathrm{a}$ & $6,95 \mathrm{ab}$ & 11,19 \\
Conversão alimentar aparente (CA) & $1,39 \mathrm{a}$ & $1,43 \mathrm{a}$ & $1,30 \mathrm{a}$ & $1,38 \mathrm{a}$ & 17,82 \\
Sobrevivência (SO) (\%) & $83,75 \mathrm{a}$ & $80,00 \mathrm{a}$ & $90,00 \mathrm{a}$ & $90,00 \mathrm{a}$ & 13 \\
\hline
\end{tabular}

Médias na mesma linha seguidas de letras distintas há diferença significativa segundo teste de Duncan $(\mathrm{P}<0,05)$. $\mathrm{CB}=$ Coração Bovino; $\mathrm{SE}$ = Sardinha Eviscerada; FP = Farinha de Peixe Chilena; FT = Filé de Tilápia.

Os melhores resultados de ganho de peso e ganho de peso diário foram observados para o tratamento com farinha de peixe não diferindo do tratamento com farinha de tilápia os piores resultados foram observados para coração bovino e sardinha eviscerada. Estes resultados são semelhantes aos constatados por Moura, Kutiza e Cyrino (2000), realizando treinamento alimentar de tucunarés (Cichla sp.), onde o ganho de peso diário variou de 0,1 a $0,17 \mathrm{~g} /$ dia, durante seus dois trabalhos analisando o treinamento alimentar. No primeiro foram analisadas duas seqüências de dietas com variação na concentração de filé de peixe moído de 80 à $0 \%$, sendo uma delas flavorizada com $10 \%$ de farinha de krill (Euphausia sp.), e no segundo trabalho utilizouse dieta inicial com $100 \%$ de filé de peixe moído sendo substituída por ração.

Kubitza (1995b) e Kubitza e Lovshin (1997b) demonstraram que uma dieta inicial com filé de peixe possibilitou os melhores resultados durante o treino alimentar do black bass, resultando em aproximadamente $75 \%$ dos peixes adaptados a ingerir uma dieta seca composta por ração para salmonídeos, em comparação com a obtenção de $41 \%$ dos peixes adaptados, pelo uso de dieta inicial composta por krill desidratado congelado (Euphausia sp.). Além disto, os alevinos alimentados inicialmente com filé de peixe, tiveram uma taxa de ganho de peso de $0,044 \mathrm{~g} / \mathrm{dia}$, contra $0,024 \mathrm{~g} /$ dia resultante dos peixes alimentados inicialmente com krill desidratado congelado.
Resultados contrários foram observados por Carneiro, Silva e Albinti (2004), os quais relatam não ter observado diferença no ganho de peso de tucunaré alimentados com dietas contendo $35 \%$ de proteína bruta, e substituída pelo microcrustáceo branchoneta (Dendrocephalus brasiliensis) seco nas proporções de 5,10 e $15 \%$.

Os resultados de transição alimentar observado no atual experimento se assemelham aos observados por Anderson (1974), Lovshin e Rushing (1989) e Willis e Flickinger $(1981,1982)$ que relatam que a transição alimentar da ração semi-úmida para ração seca foi eficaz para o "smallmouth bass". Entretanto, Fernández-Días, Pascual e Yúfera (1994) relataram que pós-larvas de "sea bream" aceitam melhor rações artificiais, quando fornecidas com alimentos vivos (náuplios de Artemia) juntamente com a ração.

A taxa de crescimento especifico é outro fator de comparação do desempenho das dietas. Os melhores resultados foram da farinha de peixe chilena $(7,29 \mathrm{~g})$ e farinha de tilápia $(6,95 \mathrm{~g})$, que demonstram sua superioridade. Portz (2001) analisando a utilização de farinha de vísceras em substituição a farinha de peixe em rações para black bass, constatou melhores resultados para a taxa de crescimento especifico $(0,91)$, em rações com $5 \%$ de farinha de peixe e $20 \%$ de farinha de vísceras, utilizando juvenis com peso médio inicial de $21 \mathrm{~g}$ durante 63 dias. Guerrero Alvarado (2003) realizando a transição alimentar de 
larvas de $P$. coruscans, a partir do alimento vivo para dieta contendo coração bovino e ração, observou valores para a taxa de crescimento especifico variando entre 2,61 e 15,83 ao longo do período experimental.

Segundo Kubitza (1995a), existem fatores de fundamental importância para o sucesso do condicionamento alimentar, como aspecto atrativo do alimento, textura adequada para a espécie, palatabilidade e estratégias de condicionamento. Vários ingredientes de origem animal como peixes moídos, subprodutos de peixes e crustáceos, órgãos de bovinos moídos, entre outros, têm sido utilizados como estimulantes, com sucesso por diversos autores (CHAITANAWISITU; MENASVETA, 1989; FLICKINGER; SMELTZER, 1983; HIGGS et al., 1985; LUZ et al., 2002; NELSON; BOWKER; ROBINSON, 1974). Kubitza e Lovshin (1997a) demonstraram que uma transição gradual dos ingredientes na dieta utilizada é mais eficiente do que uma transição gradual do alimento utilizado para o condicionamento de juvenis de black bass, a partir do "krill" desidratado e congelado para ração artificial seca.

Analisando as variáveis conversão alimentar aparente e sobrevivência, nota-se a equidade dos resultados gerados entre os tratamentos ao final do experimento. Em particular, a conversão alimentar é um forte indicativo da adaptação dos indivíduos à dieta artificial. A variável de sobrevivência demonstrou equilíbrio das condições ambientais entre os tratamentos, não interferindo no desempenho dos exemplares, visto que não houve diferença significativa constatada entre os resultados finais de cada tratamento.

Devido a isto, pressupõe-se que os melhores resultados gerados pela utilização da farinha de peixe chilena e farinha de tilápia, devem-se principalmente a maior quantidade de proteína bruta existente nestes compostos em relação aos outros utilizados, alta palatabilidade e melhor digestibilidade e equilíbrio de aminoácidos destes alimentos para esta espécie. Kubitza e Lovshin (1997b), trabalhando também com "largemouth bass", demonstram que o filé de peixe é um dos melhores atrativos alimentares para ser usado no início de dietas de treino alimentar. Portz (2001) analisado a utilização de diferentes fontes protéicas em dietas formuladas para o "black bass", constatou que as fontes protéicas de origem animal apresentaram melhores resultados para o desempenho que as fontes protéicas de origem vegetal, com melhores resultados para a farinha de vísceras de aves e farinha de peixe.

O tamanho inicial dos exemplares aliado a intensidade luminosa são dois fatores de fundamental importância para a sobrevivência, adaptação à dieta artificial e conforto dos exemplares durante o condicionamento. Segundo Luz et al. (2000) quanto mais uniforme os alevinos de espécies carnívoras, menor a taxa de canibalismo e consequentemente maior sobrevivência dos animais. Luz et al. (2002), trabalhando com condicionamento alimentar de alevinos de trairão (Hoplias cf. lacerdae), conseguiram obter $96,66 \%$ de sobrevivência, utilizando um lote homogêneo com tamanho de 2,9 90,2 cm em regime de escuridão total (0L:24D). Moura (1998) observou que alevinos de tucunaré (Cichla $\mathrm{sp)}$ com tamanhos superiores a $4 \mathrm{~cm}$ de comprimento aceitaram melhor alimentos inertes que alevinos menores. Guerrero Alvarado (2003) realizando a transição do alimento natural para dietas artificiais em larvas de $P$. coruscans, observou que a taxa de sobrevivência e aceitação ao alimento inerte aumenta quando as pós-larvas são maiores. Kubitza e Lovshin (1997b), em trabalhos com M. salmoides, obtiveram maior sucesso com a técnica de treino alimentar entre alevinos de peso inicial de 1,4 g, quando comparados com alevinos de $0,2 \mathrm{~g}$. Portanto, o tamanho inicial dos peixes utilizados no presente experimento pode ser considerado adequado para o sucesso no treinamento alimentar.

Ao final do experimento alimentavam-se da dieta artificial $100 \%$ dos indivíduos em todos os tratamentos, demonstrando a eficiência do condicionamento alimentar de alevinos de black bass, utilizando os patês protéicos testados neste trabalho. Além da eficiência da técnica utilizada, outro fator 
pode facilitar o condicionamento alimentar dos exemplares utilizados. Segundo estudos de Tidwell, Coyle e Woods (2000), as proles de alevinos de peixes oriundas de segundas ou terceiras gerações de peixes adaptados à dieta artificial, podem ser treinados mais facilmente do que proles oriundas de peixes selvagens, ou ainda, alimentados com espécies forrageiras, fato este que indica uma certa tendência à domesticação. Cavero et al. (2003), analisando o condicionamento alimentar de alevinos de pirarucu (Arapaima gigas), obtiveram $99 \%$ dos animais adaptados à dieta composta por ração, utilizando juvenis de pirarucu com peso médio de $1,5 \mathrm{~g}$ e oriundos de produção em cativeiro no estado do Amazonas. Cyrino e Kubitza (2003), trabalhando com o condicionamento alimentar de alevinos de tucunaré (Cichla $s p$ ) oriundos do ambiente natural, notaram que não houve adaptação dos peixes à ração artificial seca e isenta de atrator de alta palatabilidade após o condicionamento, aceitando o alimento inerte apenas enquanto os níveis de filé de peixe moído misturados à ração mantiveram-se acima de $40 \%$. Em todos estes trabalhos, notou-se que a eficiência do treinamento alimentar é diretamente proporcional à quantidade de atrator de alta palabilidade misturado à ração.

Durante o experimento, observaram-se algumas particularidades quanto ao canibalismo, agressões e adaptação dos indivíduos à dieta artificial. Nos tratamentos com farinha de peixes chilena e farinha de tilápia, os alevinos atacavam os péletes úmidos de patê protéico a partir do segundo dia do experimento, sendo que ao quarto dia, em todos os tratamentos, os alevinos de black bass atacavam e ingeriam os péletes antes deles chegarem ao fundo da caixa. Porém, os péletes de ração composta que tocavam o fundo da caixa, não eram ingeridos nem atacados pelos peixes, e sim, somente durante sua submersão ou ainda, quando se encontravam flutuando na superfície d'água. A partir do $12^{\circ}$ dia do experimento, notou-se que em certas unidades experimentais onde havia um número menor de exemplares devido a mortalidade, houve a ocorrência de agressões a indivíduos da réplica, e estes por sua vez, acabavam sendo mortos em conseqüência destes ataques, mas não evidenciou-se qualquer tipo de canibalismo entre os exemplares. Contudo, ao final do condicionamento alimentar, $100 \%$ dos exemplares estavam adaptados à dieta artificial totalmente seca e isenta de qualquer tipo de composto atrativo de alta palatabilidade.

\section{Conclusão}

O condicionamento alimentar utilizado no treinamento alimentar do black bass (M. salmoides) é eficaz para esta espécie carnívora. Conclui-se que as farinhas de peixes são indicadas para o treinamento alimentar, pois proporcionaram os melhores resultados.

\section{Referências}

ANDERSON, R. J. Feeding artificial diets to smallmouth bass. Progressive Fish-Culturist, Bethesda, v. 36, p. 145$151,1974$.

BOSCOLO, W. R.; HAYASHI, C.; MEURER, F. Digestibilidade aparente da energia e nutrientes de alimentos convencionais e alternativos para a tilápia do Nilo (Oreochromis niloticus, L.). Revista Brasileira de Zootecnia, Viçosa, v. 13, n. 2, p. 539-545, 2002.

BOYD, C. E. Water quality in ponds for aquaculture. Alabama: Auburn University, Birmingham Publishing, 1990.

CARNEIRO, R. L.; SILVA, J. A. M.; ALBINTI, R. C. B. Uso do microcrustáceo branchoneta (Dendrocephalus brasilensis) na ração de tucunaré. Revista Brasileira de Saúde e Produção Animal, Bahia, v. 5, n. 1, p. 18-24, 2004.

CAVERO, B. A. S.; ITUASSU, D. R. O.; PEREIRA-FILHO, M.; ROUBACH, R.; BORDINHON, A. M.; FONSECA, F. A. L.; ONO, E. A. Uso de alimento vivo como dieta inicial no treinamento alimentar de juvenis de pirarucu. Pesquisa Agropecuária Brasileira, Brasília, v. 38, n. 8, p. 1011-1015, ago. 2003.

CHAITANAWISITU, N.; MENASVETA, P. Effect of pelleted diets containing different moisture content on growth and feed conversion efficiency of juvenile seabass (Lates calcarifer BLOACH). Journal of Aquaculture Tropical, Germantown, v. 4, p. 147-155, 1989. 
CYRINO, J. E. P.; KUBITZA, F. Diets for feed training of peacock bass (Cichla sp.). Revista Scientia Agricola, Piracicaba, v. 60, n. 4, p. 609-613, Oct./Dec. 2003.

DAVIS, J. T.; LOCK, J. T. Largemouth Bass: Biology and life history. Southern Regional Aquaculture Center from United States, Department of Agriculture (SRAC). Publication nº 200, Aug. 1997.

EL-SAYED, A. F. M. Alternative dietary protein sources for farmed tilapia, Oreochromis spp. Aquaculture, Amsterdam, v. 179, n. 1, p. 149-168. 1999.

FERNÁNDEZ-DÍAZ, C.; PASCUAL, E.; YÚFERA, M. Feeding behaviour and prey size selection of gilthead sea bream, Sparus aurata, larvae fed on inert and live food. Marine Biology, Berlin, v. 118, n. 2, p. 323-328, 1994.

FLICKINGER, S. A.; SMELTZER, J. F. Training spotted bass to accept a prepared diet. Progressive Fish-Culturist, Bethesda, v. 45, n. 3, p. 185-186, 1983.

GUERRERO ALVARADO, C. E. Treinamento alimentar de pintado Pseudoplatystoma coruscans (Agassiz, 1829): Sobrevivência, crescimento e aspectos econômicos. 2003. Dissertação de (Mestrado em Aqüicultura) - Universidade Estadual Paulista, Jaboticabal.

HIGGS, D. A.; MARKERT, J. R.; PLOTNIKOFF, M. D.; MCBRIDE, J. R.; DOSANJH, B. S. Development of nutritional and environmental strategies for maximizing the growth and survival of juvenile pink salmon (Oncorhynchus gorbuscha). Aquaculture, Amsterdam, v. 47,p. 113-130, 1985.

KUBITZA, F.; LOVSHIN, L. L.; LOVELL, R. T. Identification of feed enhancers for juvenile largemouth bass Micropterus salmoides. Aquaculture, Amsterdam, v. 148, p. 191-200, 1997.

KUBITZA, F., Preparo de rações e estratégias de alimentação no cultivo intensivo de peixes carnívoros. In: SIMPÓSIO INTERNACIONAL SOBRE NUTRIÇÃO DE PEIXESECRUSTÁCEOS DO COLÉGIO BRASILEIRODE NUTRIÇÃO ANIMAL. 1995, Campos do Jordão. Anais... Campinas: CBNA, 1995a.p. 91-115.

- Intensive culture of largemouth bass Micropterus salmoides: Production of advanced juveniles and food-size fish. 1995b. Dissertation (Ph.D) - Department of Fisheries and Allied Aquacultures, Auburn University, Alabama.

KUBITZA, F.; LOVSHIN, L. L. The use of freeze-dried krill to feed train largemouth bass (Micropterus salmoides): feeds and training strategies. Aquaculture, Amsterdam, v. 148, n. 4, p. 299-312, 1997a.
Effects of initial weight and genetic strain on feed training largemouth bass Micropterus salmoides using ground fish flesh and freeze dried krill as starter diets. Aquaculture, Amsterdam, v. 148, n. 2-3, p. 179-190, 1997b.

LEYVA, G. A. Avances en el cultivo comercial de lobina (Micropterus salmoides). Panorama Acuicola, México, v. 3, n. 5, p. 10, 1998.

LOVSHIN, L. L.; RUSHING, J. H. Acceptance by largemouth bass fingerlings of pelleted feed with a gustatory additive. Progressive Fish Culturist, Bethesda, v. 51, n. 2, p. 73-78, 1989.

LUZ, R. K.; SALARO, A. L.; SOUTO, E. F.; OKANO, W. Y.; LIMA, R. R. Condicionamento alimentar de alevinos de trairão (Hoplias cf. lacerdae). Revista Brasileira de Zootecnia, Viçosa, v. 31, n. 5, p. 1881-1885, 2002.

LUZ, R. K.; SALARO, A. L.; SOUTO, E. F.; ZANIBONIFILHO, E. Avaliação de canibalismo e comportamento territorial de alevinos de trairão (Hoplias lacerdae). Acta Scientiarum, Maringá, v. 22, n. 2, p. 465-469, 2000.

MOURA, M. A. M.; KUTIZA, F.; CYRINO, J. E. D. Feed training of peacock bass (Cichla sp.). Revista Brasileira de Biologia, Rio de Janeiro, v.60, n.4, p.645-654, 2000.

MOURA, M. A. M. Estratégias de condicionamento alimentar do tucunaré (Cichla sp). 1998. Dissertação (Mestrado em Agronomia) - Escola Superior de Agricultura Luiz de Queiroz, Piracicaba.

NELSON, J. T.; BOWKER, R. G.; ROBINSON, J. D. Rearing pellet-feed largemouth bass in a raceway. Progressive Fish-Culturist, Bethesda, v. 36, n. 2, p. 108-110, 1974.

PORTZ, L. Utilização de diferentes fontes protéicas em dietas formuladas pelo conceito de proteína ideal para o "black bass" (micropterus salmoides). 2001. Tese (Doutorado em Ciência Animal e Pastagens) - Escola Superior de Agricultura Luis de Queiroz, Universidade de São Paulo, Piracicaba.

SOMMER, W. A. Um modelo CAQ/CAMpara autogestão no processo de enlatamento de sardinhas. 1998. Tese (Doutorado em Engenharia de Produção) - Universidade Federal de Santa Catarina, Florianópolis.

STOREBAKKEN, T. Krill as a potencial feed source for salmoids. Aquaculture, Amsterdam, v. 70, n. 3, p. 193-205, 1998.

TACON, G. J.; DOMINI, W. G. Overview of world aquaculture and aquafeed production. In: WORLD OF AQUACULTURE, 26., Sidney, 1999. Proceedings... Baton Rouge: World Aquaculture Society, 1999. p. 853. 
TIDWELL, J. H.; COYLE, S. D.; WOODS, T. A. Species profile: Largemouth Bass. Texas: Southern Regional Aquaculture Center from United States, Department of Agriculture (SRAC), 2000. Publication n. 722

UNIVERSIDADE FEDERAL DE VIÇOSA - UFV. SAEG: Sistema para análises estatísticas e genéticas. Versão7.1. Viçosa: UFV, 1997. Manual do usuário.
WILLIS, D. W.; FLICKINGER, S. A. Common carp eggs as a starter diets for the intensive culture of smallmouth bass fry. Progressive Fish-Culturist, Bethesda, v. 44, n. 1, p. 28-29, 1982.

WILLIS, D. W.; FLICKINGER, S. A. Intensive culture of largemouth bass fry. Transactions of the American Society of Agricultural Engineering, Saint Joseph, v. 110, p. 650$655,1981$. 